\title{
CP even and odd Higgs boson production in electron-photon collisions
}

\author{
Ken Sasaki \\ Dept. of Physics, Faculty of Engineering, Yokohama National University \\ Yokohama 240-8501, Japan \\ E-mail: sasaki@ynu.ac.jp \\ Tsuneo Uematsu* \\ Graduate School of Science, Kyoto University, Kitashirakawa, Sakyo-ku \\ Kyoto 606-8502, Japan \\ E-mail: uematsuescphys.kyoto-u.ac.jp
}

\begin{abstract}
We investigate the $\mathrm{CP}$ even and odd Higgs boson production in electron-photon collisions within the framework of standard model (SM) as well as the type-II two Higgs doublet model (2HDM) which includes the minimal supersymmetric standard model (MSSM). We evaluate the scattering amplitude for the $e \gamma$ Higgs boson production at the electroweak one-loop level. The transition form factor of the Higgs boson is introduced. In the case of the SM Higgs boson production, the $W$-boson loop contribution is dominant over that of the top-quark, while in the case of the CP-odd Higgs boson $\left(A^{0}\right)$ the dominant contribution comes from the top-quark loop, because of the absence of the direct coupling of $A^{0}$ to $W$ and $Z$ boson pairs. We reexamine the possible extra contributions to the $A^{0}$ production in the MSSM. Some arguments are given toward the analysis of CP-even Higgs production for the MSSM.
\end{abstract}

14th International Symposium on Radiative Corrections (RADCOR2019)

9-13 September 2019

Palais des Papes, Avignon, France

${ }^{*}$ Speaker. 


\section{Introduction}

The Higgs boson with mass about $125 \mathrm{GeV}$ was discovered by ATLAS and CMS at LHC [1] and its spin, parity and couplings were examined [2]. Now it would be intriguing to study its nature in $e^{+} e^{-}$collisions provided by linear colliders [3]. Along with $e^{+} e^{-}$collider, other options such as $e^{-} e^{-}, e^{-} \gamma$ and $\gamma \gamma$ colliders have also been discussed. In our previous works we have investigated the production of the SM Higgs boson $\left(H_{\mathrm{SM}}\right)[4,5]$ and the CP-odd Higgs boson $\left(A^{0}\right)[6]$ in $e \gamma$ collisions at the one-loop level in the electroweak interaction (Fig.1), where $A^{0}$ boson appears in the 2HDM or in the MSSM. We found that, for the $H_{\mathrm{SM}}$ production, the $W$-boson loops make the dominant contribution, while in the case of the $A^{0}$ production the main contribution comes from the top-quark triangle loops due to the absence of the tree-level $W^{+} W^{-} A^{0}$ and $Z Z A^{0}$ couplings. In this talk we briefly summarize the $H_{\mathrm{SM}}$ production in $e \gamma$ collisions, focusing on the transition form factor of $H_{\mathrm{SM}}$ boson, and then, we revisit the CP-odd $A^{0}$ production and look into possible extra contributions to the process in the MSSM.

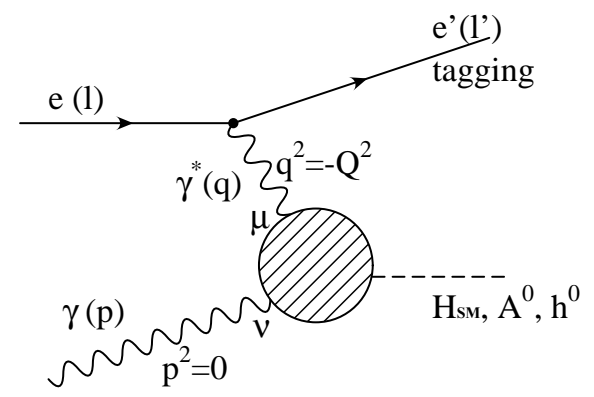

Figure 1: SM Higgs, CP-odd and CP-even Higgs boson production in $e \gamma$ collision

The loop diagrams which involve charginos and neutralinos may be important for the $A^{0}$ production in the MSSM. We examine the contributions of the chargino triangle loop diagrams and the box diagrams (i) with chargino and sneutrino and (ii) with neutralino and selectron.

\section{SM Higgs Boson Production in $e \gamma$ collisions}

The $H_{\mathrm{SM}}$ production via two-photon fusion process in $e^{+} e^{-}$or $e \gamma$ collisions has been studied as an opposite process of diphoton decay of the Higgs boson observed at LHC. In the fusion process $\gamma^{*} \gamma \rightarrow H_{\mathrm{SM}}$ in $e \gamma$ collisions, we can measure the so-called "transition form factor" of $H_{\mathrm{SM}}$ as a function of the virtual photon mass squared $\left(Q^{2}\right)$. In fact, the $\gamma^{*} \gamma \rightarrow \pi^{0}$ transition form factor has been experimentally studied in $e^{+} e^{-}$collisions by BaBar at SLAC [7] and by Belle at KEK [8], which would provide the information of the interaction and structure of the pion. In analogy with the case of pion we have investigated the transition form factor of the $H_{\mathrm{SM}}$ boson in $e \gamma$ collisions with a particular interest in its $Q^{2}$ dependence.

The scattering amplitude for $e(l)+\gamma(p) \rightarrow e^{\prime}\left(l^{\prime}\right)+H_{\mathrm{SM}}(q+p)$ through the $\gamma^{*}-\gamma$ fusion process shown in Fig. 1 is given by

$$
\left\langle e^{\prime} H|T| e \gamma\right\rangle=\bar{u}\left(l^{\prime}\right)\left(-i e \gamma_{\mu}\right) u(l) \frac{-i}{q^{2}+i \varepsilon} A^{\mu v} \varepsilon_{v}(p),
$$


where $\varepsilon_{v}(p)$ is the polarization vector of the incident real photon. Here we have introduced the tensor $A^{\mu v}$ which can be decomposed due to gauge invariance as

$$
A^{\mu v}(q, p)=\left[g^{\mu v}(q \cdot p)-p^{\mu} q^{v}\right] S_{1}\left(m^{2}, Q^{2}, m_{H}^{2}\right)+\left[q^{\mu} p^{v}-\frac{q^{2}}{q \cdot p} p^{\mu} p^{v}\right] S_{2}\left(m^{2}, Q^{2}, m_{H}^{2}\right),
$$

where $q^{2}=-Q^{2}<0, p^{2}=0$ and $(q+p)^{2}=p_{H}^{2}=m_{H}^{2}$. We denote collectively the mass of the intermediate particle in the loop by $m$. The first factor $S_{1}$ leads to the transition form factor while the second factor $S_{2}$ does not contribute to the scattering amplitude since $p^{v} \varepsilon_{v}(p)=0$.

We define the transition form factor $F_{i}\left(m^{2}, Q^{2}, m_{H}^{2}\right)$ by

$$
F_{i}\left(m^{2}, Q^{2}, m_{H}^{2}\right)=S_{1}\left(m^{2}, Q^{2}, m_{H}^{2}\right) /\left(\frac{g e^{2}}{(4 \pi)^{2}} \frac{1}{m_{W}}\right)
$$

where $i=1 / 2,1$ for a fermion-loop $F_{1 / 2}$ and for the $W$-boson loop $F_{1}$, respectively. $e$ and $g$ are the electromagnetic and weak gauge couplings, respectively, and $m_{W}$ is the $W$ boson mass.

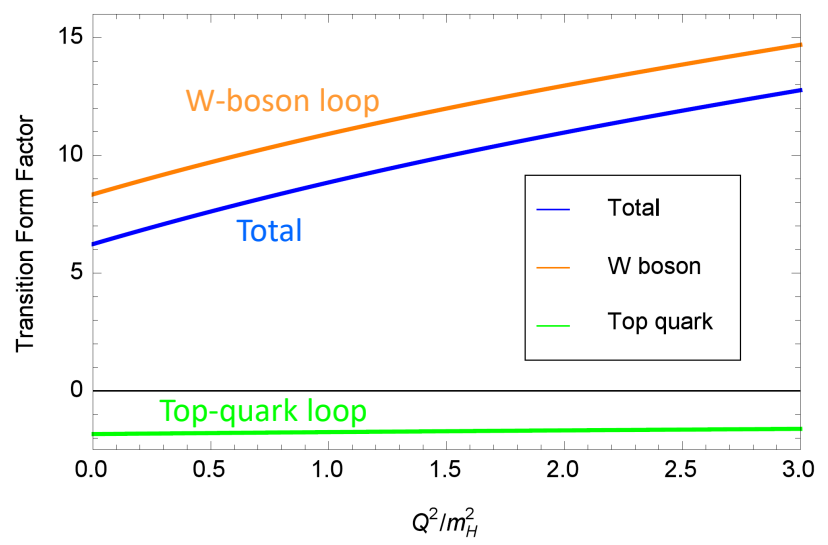

Figure 2: Transition form factor of the Higgs boson as a function of $Q^{2}$ in units of $m_{H}^{2}$. The $W$-boson contribution is dominant over that of the top-quark. The blue line shows the total contribution.

The total transition form factor is given by

$$
F_{\text {total }}\left(Q^{2}, m_{H}^{2}\right)=\sum_{f} N_{c} e_{f}^{2} F_{1 / 2}\left(m_{f}^{2}, Q^{2}, m_{H}^{2}\right)+F_{1}\left(m_{W}^{2}, Q^{2}, m_{H}^{2}\right),
$$

where $N_{c}$ is the number of the colors ( 1 for leptons and 3 for quarks) and $e_{f}$ is the electric charge of the fermion $f$ in the unit of proton charge. We found that at the one-loop level the $W$ boson loop contribution dominates over that of the top-quark as shown in Fig.2. Note that we have to consider the $Z^{*} \gamma$ fusion process which cannot be ignored for the large $Q^{2}$ region [4, 5].

\section{CP-odd Higgs Boson Production in $e \gamma$ collisions in Type-II 2HDM}

As a minimal extension of the Higgs sector of the SM, we consider the type-II 2HDM which includes the MSSM as a special case [9]. After the spontaneous symmetry breaking, there appear 
two charged and three neutral Higgs bosons:

$$
\text { Charged } \quad H^{+}, H^{-} ; \quad \text { CP-even } \quad h^{0}, H^{0} ; \quad \text { CP-odd } \quad A^{0} .
$$

Here we focus on the CP-odd Higgs boson $A^{0}$, and investigate its transition form factor obtained in the $\gamma^{*}-\gamma$ fusion process in $e \gamma$ collisions.

The characteristics of $A^{0}$ couplings to other fields in the type-II 2HDM are the following: 1) In contrast to the CP-even Higgs bosons $h^{0}$ and $H^{0}, A^{0}$ does not couple to $W^{+} W^{-}$and $Z Z$ pairs at tree level. Hence $W$-boson and $Z$-boson one-loop diagrams do not contribute to the $A^{0}$ production. 2) $A^{0}$ does not couple to other two physical Higgs bosons in cubic interactions. 3) The couplings of $A^{0}$ to the fermions are proportional to the fermion masses. Therefore, we only consider the top quark for the charged fermion loop diagrams. The $A^{0}$ coupling to the top quark with mass $m_{t}$ is given by $\lambda \gamma_{5}$ with $\lambda=-g m_{t} \cot \beta /\left(2 m_{W}\right)$, where $\cot \beta=\left\langle H_{1}\right\rangle /\left\langle H_{2}\right\rangle$, the ratio of the vacuum expectation values of two Higgs bosons [9]. The scattering amplitude for $e(l)+\gamma(p) \rightarrow e^{\prime}\left(l^{\prime}\right)+A^{0}\left(p_{A}\right)$ through

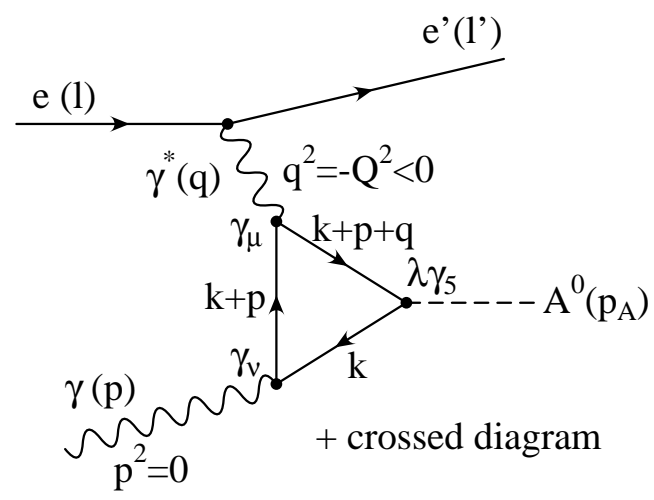

Figure 3: Top-quark triangle loop $\gamma^{*} \gamma$ fusion diagram for $e \gamma \rightarrow e^{\prime} A^{0}$

the $\gamma^{*}-\gamma$ fusion process is given by

$$
\left\langle e^{\prime} A^{0}|T| e \gamma\right\rangle_{\gamma^{*} \gamma}=\bar{u}\left(l^{\prime}\right)\left(-i e \gamma_{\mu}\right) u(l) \frac{-i}{q^{2}+i \varepsilon} \widetilde{A}^{\mu v} \varepsilon_{v}(p) .
$$

The structure tensor $\widetilde{A}^{\mu v}$ is expressed as

$$
\widetilde{A}^{\mu v}(q, p)=\frac{g e^{2}}{(4 \pi)^{2}} \frac{1}{m_{W}} \widetilde{F}\left(m_{t}^{2}, Q^{2}, m_{A}^{2}\right) \varepsilon^{\mu v \alpha \beta} q_{\alpha} p_{\beta},
$$

where we have introduced the transition form factor $\widetilde{F}$ as

$$
\widetilde{F}\left(m_{t}^{2}, Q^{2}, m_{A}^{2}\right) \equiv-N_{c} e_{t}^{2} \cot \beta 4 m_{t}^{2} C_{0}\left(0,-Q^{2}, m_{A}^{2}, m_{t}^{2}, m_{t}^{2}, m_{t}^{2}\right),
$$

and $C_{0}$ is a Passarino-Veltman's scalar integral for three-point functions [10].

\section{Extra Contributions to the $A^{0}$ production in MSSM - I}

In the MSSM, the supersymmetric particles, such as squarks, charginos and neutralinos, may come into play for the $A^{0}$ production in $e \gamma$ collisions. 


\subsection{Trilinear $A^{0}$ Coupling to Squark Pairs}

We notice that squark-pairs of the mass eigenstates do not couple to the CP-odd Higgs boson $A^{0}$ as shown in Fig. 4 since the coupling is antisymmetric under the exchange of the indices $i, j$ of the mass eigenstates. Namely, for $i=j$ the above coupling vanishes [9]. Hence, the scalar top-quark (stop) does not contribute to the $A^{0}$ production in $e \gamma$ collisions at one-loop level.

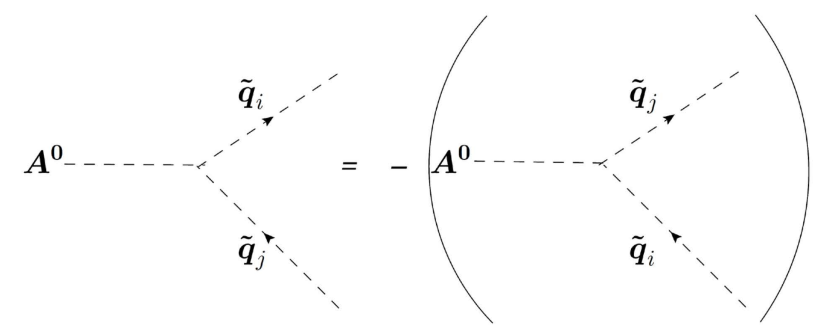

Figure 4: Coupling of squark mass eigenstates $\tilde{q}_{i}(i=1,2)$ to CP-odd Higgs Boson $A^{0}$

\subsection{Chargino Contributions to Transition Form Factor}

The contributions from charginos $\tilde{\chi}_{i}^{ \pm}(\mathrm{i}=1,2)$ are obtained by replacing the top-quark triangle loop diagrams with the chargino counterparts as shown in Fig.3. The couplings of $A^{0}$ to the chargino mass eigenstates are purely pseudoscalar and are expressed as $g \kappa_{i} \gamma_{5}$. We assume $\kappa_{i} \sim \mathscr{O}(1)$ and take $m_{\chi_{i}}=1 \mathrm{TeV}$ as a benchmark mass for charginos. Then, for $\tan \beta \leq 10$, it turns out that the charginos do not give a sizable contribution. See Fig.5.

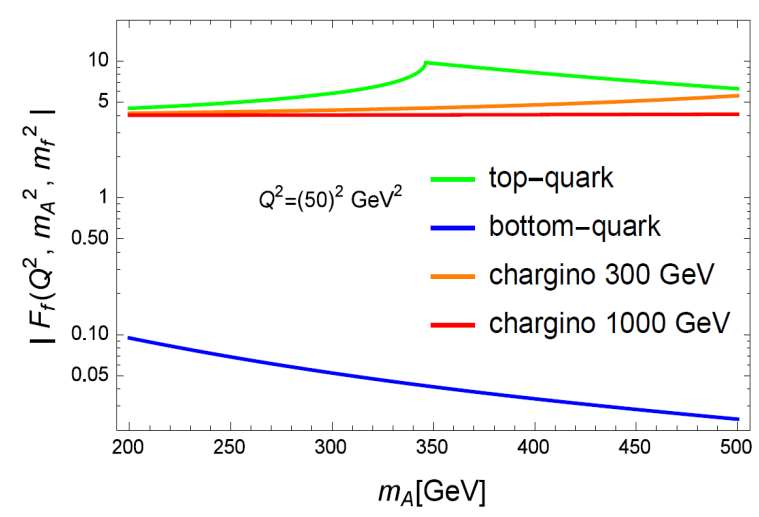

Figure 5: The transition form factors of $A^{0}$ as a function of $m_{A}$ with $Q^{2}=(50)^{2} \mathrm{GeV}^{2}$ for the chargino mass $300 \mathrm{GeV}^{2}$ and $1000 \mathrm{GeV}^{2}$ together with top-quark and bottom-quark loops. 


\section{Extra Contributions to the $A^{0}$ production in MSSM - II}

Next we consider one-loop box diagrams shown in Fig.6 involving charginos $\tilde{\chi}_{i}^{ \pm}(i=1,2)$, sneutrino $\tilde{v}$, neutralinos $\tilde{\chi}_{i}^{0}(i=1,2,3,4)$ and selectron $\tilde{e}_{i}(i=1,2)$ appearing in the MSSM which are relevant for the $A^{0}$ production in $e \gamma$ collisions. Since we are only interested in the order of magnitude for the production cross section, we assume that only mass eigenstates $\tilde{\chi}_{1}^{ \pm}, \tilde{\chi}_{1}^{0}$ and $\tilde{e}_{1}$ contribute dominantly to the reactions.

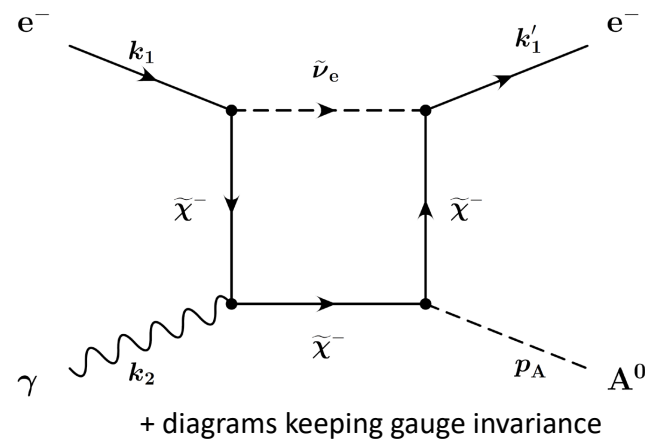

(a)

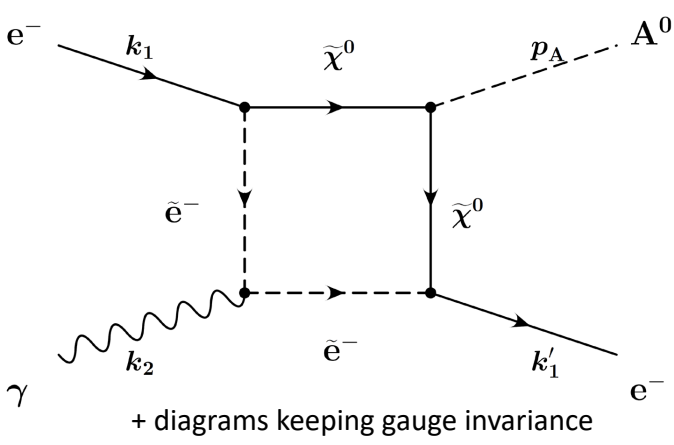

(b)

Figure 6: (a) Chargino-sneutrino-Box contribution

(b) Neutralino-selectron-Box contribution

\subsection{Chargino-sneutrino-box contribution}

The interaction Lagrangian for the vertices $A^{0} \bar{\chi}_{1} \tilde{\chi}_{1}$ (see Fig. A.24(c) of [9]) and $e \tilde{\chi}_{1} \tilde{v}$ (see Eq.(C30) of [11]) are given, respectively, by

$$
\mathscr{L}_{A^{0} \overline{\tilde{\chi}} \tilde{\chi}}=-i g \kappa_{1} \overline{\widetilde{\chi}}_{1} \gamma_{5} \widetilde{\chi}_{1} A^{0}, \quad \quad \mathscr{L}_{\tilde{\chi} e \tilde{v}}=-g V_{11}^{*} \overline{\widetilde{\chi}_{1}^{c}} P_{\mathrm{L}} e \widetilde{v}^{*}+h . c .
$$

The one-loop amplitude for $e^{-}\left(k_{1}\right)+\gamma\left(k_{2}\right) \rightarrow e^{-}\left(k_{1}^{\prime}\right)+A^{0}\left(p_{A}\right)$, shown in Fig.6(a), is found to be

$$
A_{e \gamma \rightarrow e A^{0}}^{(\tilde{\chi} \tilde{v})}=\left(\frac{e g^{3} \kappa_{1}\left|V_{11}\right|^{2}}{16 \pi^{2}}\right) \frac{m_{\tilde{\chi}}}{4}\left[\bar{u}\left(k_{1}^{\prime}\right) F_{(\tilde{\chi} \tilde{v}) \beta}\left(1-\gamma_{5}\right) u\left(k_{1}\right)\right] \varepsilon\left(k_{2}\right)^{\beta}
$$

where the factor $\left(1-\gamma_{5}\right)$ is due to the $e-\widetilde{\chi}-\widetilde{v}$ vertex. We perform gauge invariant decomposition of the amplitude as

$$
F_{(\tilde{\chi} \tilde{v}) \beta}=\left(\frac{2 k_{1 \beta} k_{2}}{s}-\gamma_{\beta}\right) S_{\left(k_{1}\right)}^{\tilde{\chi} \tilde{v}}\left(s, t, m_{A^{0}}^{2}, m_{\tilde{\chi}}^{2}, m_{\tilde{v}}^{2}\right)+\left(\frac{2 k_{1 \beta}^{\prime} k_{2}}{u}+\gamma_{\beta}\right) S_{\left(k_{1}^{\prime}\right)}^{\tilde{\chi} \tilde{v}}\left(s, t, m_{A^{0}}^{2}, m_{\tilde{\chi}}^{2}, m_{\tilde{v}}^{2}\right),
$$

where $S_{\left(k_{1}\right)}^{\tilde{\chi} \tilde{v}}$ and $S_{\left(k_{1}^{\prime}\right)}^{\tilde{\tilde{\chi} \tilde{v}}}$ are expressed in terms of Passarino-Veltman's scalar integrals [10], $B_{0}$ 's, $C_{0}$ 's and $D_{0}$ 's, as follows:

$$
S_{\left(k_{1}\right)}^{\tilde{\chi} \tilde{v}}\left(s, t, m_{A^{0}}^{2}, m_{\tilde{\chi}}^{2}, m_{\tilde{v}}^{2}\right)
$$




$$
\begin{aligned}
& =-\frac{s-t}{t} C_{0}\left(0, u, m_{A^{0}}^{2}, m_{\tilde{\chi}}^{2}, m_{\tilde{v}}^{2}, m_{\tilde{\chi}}^{2}\right)-\frac{u}{t} C_{0}\left(0,0, u, m_{\tilde{\chi}}^{2}, m_{\tilde{\chi}}^{2}, m_{\tilde{v}}^{2}\right)+\frac{2(s+u)}{t} C_{0}\left(0, t, m_{A^{0}}^{2}, m_{\tilde{\chi}}^{2}, m_{\tilde{\chi}}^{2}, m_{\tilde{\chi}}^{2}\right) \\
& -\frac{\left(m_{\tilde{\chi}}^{2}(s+u)-m_{\tilde{\nu}}^{2}(s+u)-t u\right)}{t} D_{0}\left(0,0,0, m_{A^{0}}^{2}, t, u, m_{\tilde{\chi}}^{2}, m_{\tilde{v}}^{2}, m_{\tilde{\chi}}^{2}, m_{\tilde{\chi}}^{2}\right)-\frac{t+u}{t} C_{0}\left(0, s, m_{A^{0}}^{2}, m_{\tilde{\chi}}^{2}, m_{\tilde{v}}^{2}, m_{\tilde{\chi}}^{2}\right) \\
& -{ }_{t}^{s} C_{0}\left(0,0, s, m_{\tilde{\chi}}^{2}, m_{\tilde{\chi}}^{2}, m_{\tilde{v}}^{2}\right)-\frac{\left(m_{\tilde{\chi}}^{2}(s+u)-m_{\tilde{v}}^{2}(s+u)+s t\right)}{t} D_{0}\left(0,0,0, m_{A^{0}}^{2}, s, t, m_{\tilde{\chi}}^{2}, m_{\tilde{\chi}}^{2}, m_{\tilde{v}}^{2}, m_{\tilde{\chi}}^{2}\right),(5.4)
\end{aligned}
$$

and

$$
\begin{aligned}
& S_{\left(k_{1}^{\prime}\right)}^{\tilde{\chi} \tilde{v}}\left(s, t, m_{A^{0}}^{2}, m_{\tilde{\chi}}^{2}, m_{\tilde{v}}^{2}\right) \\
& =-\frac{u-t}{t} C_{0}\left(0, s, m_{A^{0}}^{2}, m_{\tilde{\chi}}^{2}, m_{\tilde{v}}^{2}, m_{\tilde{\chi}}^{2}\right)-\frac{s}{t} C_{0}\left(0,0, s, m_{\tilde{\chi}}^{2}, m_{\tilde{\chi}}^{2}, m_{\tilde{v}}^{2}\right)+\frac{2(s+u)}{t} C_{0}\left(0, t, m_{A^{0}}^{2}, m_{\tilde{\chi}}^{2}, m_{\tilde{\chi}}^{2}, m_{\tilde{\chi}}^{2}\right) \\
& -\frac{\left(m_{\tilde{\chi}}^{2}(s+u)-m_{\tilde{\nu}}^{2}(s+u)-s t\right)}{t} D_{0}\left(0,0,0, m_{A^{0}}^{2}, s, t, m_{\tilde{\chi}}^{2}, m_{\tilde{\chi}}^{2}, m_{\tilde{v}}^{2}, m_{\tilde{\chi}}^{2}\right)-\frac{s+t}{t} C_{0}\left(0, u, m_{A^{0}}^{2}, m_{\tilde{\chi}}^{2}, m_{\tilde{v}}^{2}, m_{\tilde{\chi}}^{2}\right) \\
& -\frac{u}{t} C_{0}\left(0,0, u, m_{\tilde{\chi}}^{2}, m_{\tilde{\chi}}^{2}, m_{\tilde{v}}^{2}\right)-\frac{\left(m_{\tilde{\chi}}^{2}(s+u)-m_{\tilde{v}}^{2}(s+u)+t u\right)}{t} D_{0}\left(0,0,0, m_{A^{0}}^{2}, t, u, m_{\tilde{\chi}}^{2}, m_{\tilde{v}}^{2}, m_{\tilde{\chi}}^{2}, m_{\tilde{\chi}}^{2}\right)(5.5)
\end{aligned}
$$

Here we have assumed that an electron is massless and introduced the following Mandelstam variables: $s=2 k_{1} \cdot k_{2}, t \equiv-Q^{2}=-2 k_{1} \cdot k_{1}^{\prime}, u=-2 k_{2} \cdot k_{1}^{\prime}=m_{A^{0}}^{2}-s-t$ and $p_{A}^{2}=m_{A^{0}}^{2}$.

In terms of the above invariant functions the unpolarized differential cross section is given by

$$
\frac{d \sigma_{\tilde{\chi} \tilde{v}}}{d t}=\frac{1}{16 \pi s^{2}}\left(\frac{e g^{3} \kappa_{1}\left|V_{11}\right|^{2}}{16 \pi^{2}}\right)^{2} \cdot \frac{m_{\tilde{\chi}}^{2}}{8}(-t) \times\left\{\left|S_{\left(k_{1}\right)}^{\tilde{\chi} \tilde{v}}\right|^{2}+\left|S_{\left(k_{1}^{\prime}\right)}^{\tilde{\chi} \tilde{v}}\right|^{2}\right\} .
$$

We estimate the order of the cross sections with the assumption for the coupling and mixing matrix element satisfying $\kappa_{1}\left|V_{11}\right|^{2} \sim \mathscr{O}(1)$. It would be difficult to exhaust the full mass parameter space. Here we only give the case where $m_{\text {chargino }}=200$ and $1000 \mathrm{GeV}$ with $m_{\text {sneutrino }}=300 \mathrm{GeV}$. Note that the chargino-sneutrino box contribution is much smaller than the top-quark triangle-loop contribution, especially in the lower $Q^{2}$ region. See Fig.7.

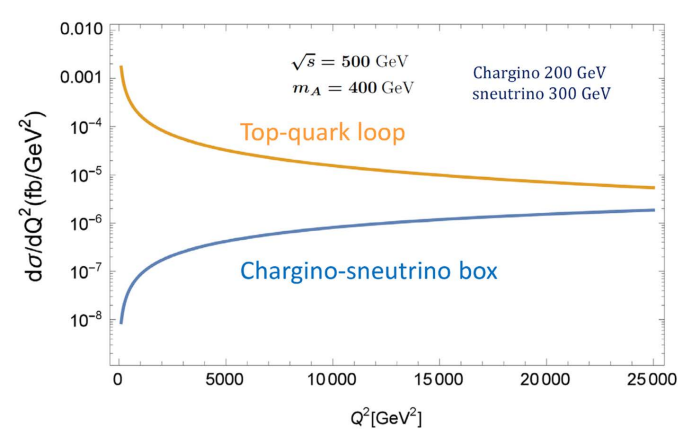

(a)

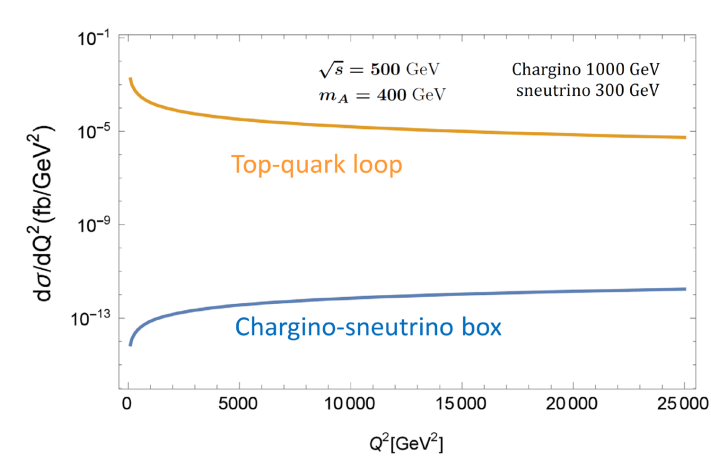

(b)

Figure 7: Top-quark loop (orange) and chargino-sneutrino (blue) contribution to $e+\gamma \rightarrow e^{\prime}+A^{0}$ production cross section. $\sqrt{s}=500 \mathrm{GeV}$ and $m_{A}=400 \mathrm{GeV}$, (a) $m_{\text {chargino }}=200 \mathrm{GeV}$ and $m_{\text {sneutrino }}=300 \mathrm{GeV}$ (b) $m_{\text {chargino }}=1000 \mathrm{GeV}$ and $m_{\text {sneutrino }}=300 \mathrm{GeV}$. 


\subsection{Neutralino-selectron-box contribution}

The neutralino-electron-selectron interaction terms are given in Eq.(C52) of [11]. We extract the relevant term for $\widetilde{\chi}_{1}^{0}-e-\widetilde{e}_{1}$, which is expressed as

$$
\mathscr{L}_{\tilde{\chi}_{1}^{0} e \tilde{e}_{1}}=g \overline{\widetilde{\chi}}_{1}^{0}\left(a+b \gamma_{5}\right) e \tilde{e}_{1}^{*}+h . c . .
$$

where $a$ and $b$ are written in terms of the matrix elements introduced to diagonalize mass matrices for neutralinos and selectrons and $g^{\prime} / g$, the ratio of the $\mathrm{U}(1)$ to $\mathrm{SU}(2)$ gauge coupling constants. The $A^{0}$ coupling to the neutralino $\tilde{\chi}_{1}^{0}$ is given by (see Fig. A.26(c) of [9]),

$$
\mathscr{L}_{A^{0} \bar{\chi}_{1}^{0} \widetilde{\chi}_{1}^{0}}=-i g \eta_{1} \overline{\widetilde{\chi}_{1}^{0}} \gamma_{5} \widetilde{\chi}_{1}^{0} A^{0}
$$

Since $\tilde{\chi}_{1}^{0}$ is a Majorana particle, we follow the Feynman rules for Majorana fermions given by Denner et al.[12].

The one-loop amplitude for $e^{-}\left(k_{1}\right)+\gamma\left(k_{2}\right) \rightarrow e^{-}\left(k_{1}^{\prime}\right)+A^{0}\left(p_{A}\right)$ through the neutralino-selectron box diagrams shown in Fig.6(b), is found to be

$$
A_{e \gamma \rightarrow e A^{0}}^{\left(\tilde{\chi}_{1} \tilde{e}_{1}\right)}=\left(\frac{i e g^{3} \eta_{1}}{16 \pi^{2}}\right)\left[\bar{u}\left(k_{1}^{\prime}\right)\left\{F_{1 \beta}^{\left(\tilde{\chi}_{1}^{0} \tilde{e}_{1}\right)} A_{1}\left(a, b, \gamma_{5}\right)+m_{\tilde{\chi}_{1}^{0}} F_{2 \beta}^{\left(\tilde{\chi}_{1}^{0} \tilde{e}_{1}\right)} A_{2}\left(a, b, \gamma_{5}\right)\right\} u\left(k_{1}\right)\right] \varepsilon\left(k_{2}\right)^{\beta}
$$

where $A_{1}\left(a, b, \gamma_{5}\right) \equiv\left(|a|^{2}-|b|^{2}\right) \gamma_{5}+\left(a^{*} b-a b^{*}\right)$, and $A_{2}\left(a, b, \gamma_{5}\right) \equiv\left(|a|^{2}+|b|^{2}\right) \gamma_{5}+\left(a^{*} b+a b^{*}\right)$. The factors $F_{1 \beta}^{\left(\tilde{\chi}_{1}^{0} \tilde{e}_{1}\right)}$ and $F_{2 \beta}^{\left(\tilde{\chi}_{1}^{0} \tilde{e}_{1}\right)}$ are written in gauge invariant forms as

$$
\begin{aligned}
& F_{1 \beta}^{\left(\tilde{\chi}_{1}^{0} \tilde{e}_{1}\right)}=k_{2} \gamma_{\beta} T_{A}\left(s, t, m_{A^{0}}^{2}, m_{\tilde{\chi}_{1}^{0}}^{2}, m_{\tilde{e}_{1}}^{2}\right)+\left(u k_{1 \beta}+s k_{1 \beta}^{\prime}\right) T_{B}\left(s, t, m_{A^{0}}^{2}, m_{\tilde{\chi}_{1}^{0}}^{2}, m_{\tilde{e}_{1}}^{2}\right), \\
& F_{2 \beta}^{\left(\tilde{\chi}_{1}^{0} \tilde{e}_{1}\right)}=\left(\frac{2 k_{1 \beta} k_{2}}{s}-\gamma_{\beta}\right) S_{\left(k_{1}\right)}^{\tilde{\chi}_{1}^{0} \tilde{e}_{1}}\left(s, t, m_{A^{0}}^{2}, m_{\tilde{\chi}_{1}^{0}}^{2}, m_{\tilde{e}_{1}}^{2}\right)+\left(\frac{2 k_{1 \beta}^{\prime} k_{2}}{u}+\gamma_{\beta}\right) S_{\left(k_{1}^{\prime}\right)}^{\tilde{\chi}_{1}^{0} \tilde{e}_{1}}\left(s, t, m_{A^{0}}^{2}, m_{\tilde{\chi}_{1}^{0}}^{2}, m_{\tilde{e}_{1}}^{2}\right),
\end{aligned}
$$

where $T_{A}, T_{B}, S_{\left(k_{1}\right)}$ and $S_{\left(k_{1}^{\prime}\right)}$ are expressed in terms of the Passarino-Veltman scalar integrals $B_{0}$ 's, $C_{0}$ 's and $D_{0}$ 's.

It would be difficult to study the behaviors of the cross section in the full parameter space. Here we consider a case where the function $A_{1}\left(a, b, \gamma_{5}\right)$ is close to zero. Assuming $A_{1}\left(a, b, \gamma_{5}\right)=0$, we have $|a|^{2}-|b|^{2}=0, a^{*} b-a b^{*}=0$, which leads to

$$
A_{2}\left(a, b, \gamma_{5}\right)= \pm 2|a|^{2}\left(1 \pm \gamma_{5}\right) \quad \text { (double-sign corresponds). }
$$

Hence the scattering amplitude is written as

$$
A_{e \gamma \rightarrow e A^{0}}^{\left(\tilde{\chi}_{1}^{0} \tilde{e}_{1}\right)}=\frac{i e g^{3} \eta_{1} \tilde{N}}{16 \pi^{2}} \frac{m_{\tilde{\chi}_{1}^{0}}}{4} \bar{u}\left(k_{1}^{\prime}\right) F_{2 \beta}^{\left(\tilde{\chi}_{1}^{0} \tilde{e}_{1}\right)}\left(1 \pm \gamma_{5}\right) u\left(k_{1}\right) \varepsilon\left(k_{2}\right)^{\beta} .
$$

where we put $\tilde{N}=\left( \pm 8|a|^{2}\right)$. The differential cross section is found to be

$$
\frac{d \sigma_{\tilde{\chi}_{1}^{0} \tilde{e}}}{d t}=\frac{1}{16 \pi s^{2}}\left(\frac{e g^{3} \eta_{1} \tilde{N}}{16 \pi^{2}}\right)^{2} \cdot \frac{m_{\tilde{\chi}_{1}^{0}}^{2}}{8}(-t) \times\left\{\left|S_{\left(k_{1}\right)}^{\tilde{\chi}_{1}^{0} \tilde{e}_{1}}\right|^{2}+\left|S_{\left(k_{1}^{\prime}\right)}^{\tilde{\chi}_{1}^{0} \tilde{e}_{1}}\right|^{2}\right\} .
$$

Assuming $\left|\eta_{1} \tilde{N}\right| \sim \mathscr{O}(1)$, we plot $d \sigma_{\tilde{\chi}_{1}^{0} \tilde{e}} / d t$ for some mass parameters in Fig.8. 


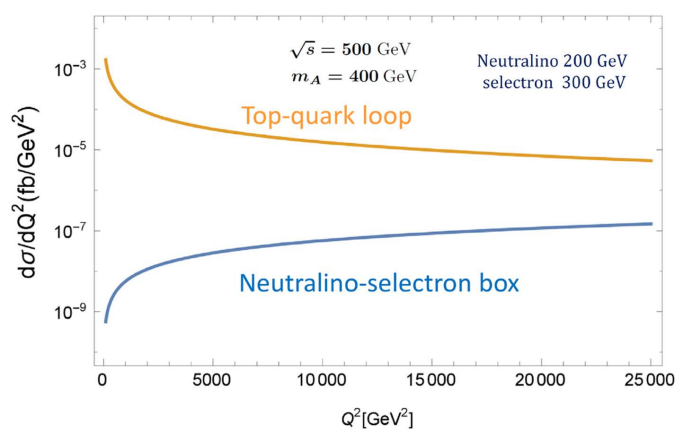

(a)

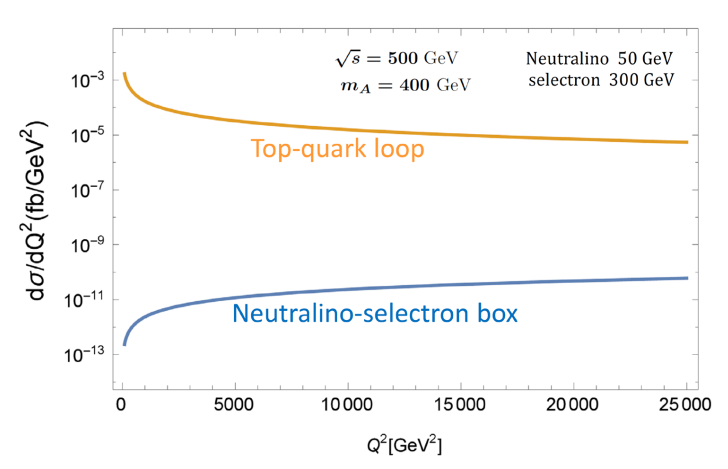

(b)

Figure 8: (a) Top-quark loop (orange) and Neutralino-selectron (blue) contribution to $e+\gamma \rightarrow e^{\prime}+A^{0}$ production cross section. $\sqrt{s}=500 \mathrm{GeV}$ and $m_{A}=400 \mathrm{GeV}$, (a) $m_{\text {neutralino }}=200 \mathrm{GeV}$ and $m_{\text {selectron }}=300$ $\mathrm{GeV}$ (b) $m_{\text {neutralino }}=50 \mathrm{GeV}$ and $m_{\text {selectron }}=300 \mathrm{GeV}$.

\section{Toward the CP-even Higgs boson $h^{0}$ production}

The above argument on the Higgs boson production in $e \gamma$ collisions, can be extended to the case of the CP-even Higgs $h^{0}$ production in 2HDM including MSSM. The possible $\gamma^{*} / Z^{*}-\gamma$ fusion diagrams contributing the $h^{0}$ production are shown in Fig.9, where we also plotted the transition form factor of $h^{0}$ production. As can be seen, contribution from the charged Higgs $H^{ \pm}$is negligibly small. The chargino-sneutrino and neutralino-selectron box diagrams should also be studied.
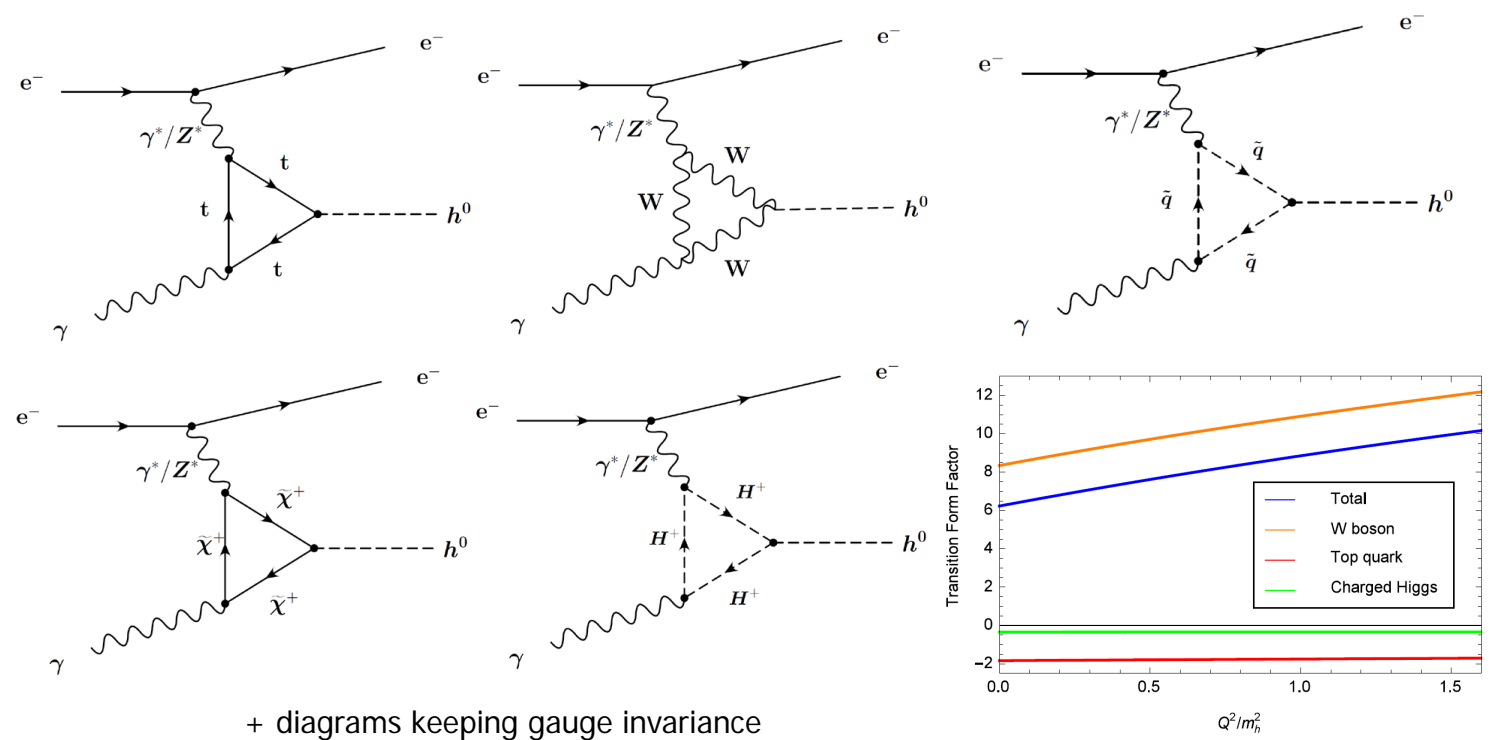

Figure 9: $\gamma^{*} / Z^{*}-\gamma$ fusion diagrams contributing to the $h^{0}$ production together with the transition form factor coming from the loops of W-boson, top-quark and charged Higgs of mass $300 \mathrm{GeV}$. 


\section{Conclusion}

In this talk we first briefly summarize what we have studied $e \gamma$ production of the SM Higgs as well as CP-odd Higgs boson appearing in type-II 2HDM including MSSM. Then we examine potentially possible extra contributions to the CP-odd Higgs production arising from the charginosneutrino and neutralino-selectron related processes. These processes do not give sizable effects in the mass parameter space where we have studied. Now let us refer to the $\gamma^{*} / Z^{*}-\gamma$ fusion diagram as "F-type"and to the Box-diagram as "B-type". Then, if the F-type diagrams are dominant over the B-type diagrams then the transition form factor provides a good description of the process, where the production cross section is proportional to the absolute square of the transition form factor. Some arguments are also given toward the analysis of CP-even Higgs boson production.

\section{Acknowledgments}

We would like to thank the organizers of the RADCOR 2019 for the hospitality at such a well-organized and stimulating symposium.

\section{References}

[1] ATLAS Collaboration, Phys. Lett. B716, 1 (2012); CMS Collaboration, Phys. Lett. B716, 30 (2012).

[2] ATLAS Collaboration, Phys. Lett. B726, 88 (2013); Phys. Lett. B726, 120 (2013); CMS

Collaboration, Phys. Rev. Lett. 110, 081803 (2013).

[3] http://www.linearcollider.org.

[4] N. Watanabe, Y. Kurihara, K. Sasaki and T. Uematsu, Phys. Lett. B728, 202 (2014); PoS (RADCOR 2013) 050; PoS (RADCOR 2013) 053; PoS (QFTHEP 2013) 040.

[5] N. Watanabe, Y. Kurihara, T. Uematsu,and K. Sasaki, Phys. Rev. D90, 033015 (2014); CERN Proc. 1 (2018) 27.

[6] K. Sasaki and T. Uematsu, Phys. Lett. B781, 290 (2018); PoS(RADCOR 2017) 066 (2017).

[7] B. Aubert et al. (BaBar Collaboration), Phys. Rev. D80 052002 (2009).

[8] S. Uehara et al. (Belle Collaboration), Phys. Rev. D86 092007 (2012).

[9] J. F. Gunion, H. E. Haber, G. Kane and S. Dawason, “The Higgs Hunter's Guide”(Addison-Wesley, 1990).

[10] G. Passarino and M. Veltman, Nucl. Phys. B160, 151 (1979); G. 't Hooft and M.J.G. Veltman, Nucl. Phys. B153, 365 (1979); G.J. van Oldenborgh and J.A.M. Vermaseren, Z. Physik C46 425 (1990).

[11] H. E. Haber and G. L. Kane, Phys. Rept. 117, 75 (1985).

[12] A. Denner, H. Eck, O. Hahn and J. Küblbeck, Nucl.Phys. B387, 467(1992). 